\title{
Searching for dark clouds in the outer galactic plane ${ }^{\star}$
}

\section{A statistical approach for identifying extended red(dened) regions in 2MASS}

\author{
W. W. F. Frieswijk ${ }^{1}$ and R. F. Shipman ${ }^{2,1}$ \\ 1 Kapteyn Astronomical Institute, University of Groningen, PO Box 800, Landleven 12, 9700 AV Groningen, The Netherlands \\ e-mail: frieswyk@astro.rug.nl \\ 2 SRON, National Institute for Space Research, PO Box 800, Landleven 12, 9700 AV Groningen, The Netherlands
}

Received 28 July 2009 / Accepted 29 January 2010

\begin{abstract}
Context. Most of what is known about clustered star formation to date comes from well studied star forming regions located relatively nearby, such as Rho-Ophiuchus, Serpens and Perseus. However, the recent discovery of infrared dark clouds may give new insights in our understanding of this dominant mode of star formation in the Galaxy. Though the exact role of infrared dark clouds in the formation process is still somewhat unclear, they seem to provide useful laboratories to study the very early stages of clustered star formation. Infrared dark clouds have been identified predominantly toward the bright inner parts of the galactic plane. The low background emission makes it more difficult to identify similar objects in mid-infrared absorption in the outer parts. This is unfortunate, because the outer Galaxy represents the only nearby region where we can study effects of different (external) conditions on the star formation process.

Aims. The aim of this paper is to identify extended red regions in the outer galactic plane based on reddening of stars in the nearinfrared. We argue that these regions appear reddened mainly due to extinction caused by molecular clouds and young stellar objects. The work presented here is used as a basis for identifying star forming regions and in particular the very early stages. An accompanying paper describes the cross-identification of the identified regions with existing data, uncovering more on the nature of the reddening. Methods. We use the Mann-Whitney U-test, in combination with a friends-of-friends algorithm, to identify extended reddened regions in the 2MASS all-sky JHK survey. We process the data on a regular grid using two different resolutions, 60" and 90". The two resolutions have been chosen because the stellar surface density varies between the crowded spiral arm regions and the sparsely populated galactic anti-center region.

Results. We identify 1320 extended red regions at the higher resolution and 1589 in the lower resolution run. The linear extent of the identified regions ranges from a few arc-minutes to about a degree.

Conclusions. The majority of extended red regions are associated with major molecular cloud complexes, supporting our hypothesis that the reddening is mostly due to foreground clouds and embedded objects. The reliability of the identified regions is $>99.9 \%$. Because we choose to identify object with a high reliability we can not quantify the completeness of the list of regions.
\end{abstract}

Key words. methods: statistical - catalogs - stars: formation - ISM: clouds - dust, extinction

\section{Introduction}

Dark clouds represent the earliest stages of star formation in the Galaxy. Nearby dark clouds have been studied in great detail and provide a wealth of information on the low-mass isolated star formation process (e.g., Shu et al. 1987). However, most stars do not form in isolation but in groups or clusters (Lada \& Lada 2003), and this clustered mode of star formation is not well understood. Some key questions of current star formation research are the origin of the stellar mass distribution, the relation between high-mass star formation and stellar clusters and the effect of environment on the star formation process. See Zinnecker \& Yorke (2007) for a recent review of this topic.

In the mid $1990 \mathrm{~s}$, infrared surveys with the infrared space observatory (ISO) and the Midcourse Space Experiment (MSX) have revealed a class of dark clouds, referred to as infrared dark

* Full Table 1 is only available in electronic form at the CDS via anonymous ftp to cdsarc.u-strasbg.fr $(130.79 .128 .5)$ or via http://cdsweb.u-strasbg.fr/cgi-bin/qcat?J/A+A/515/A51 clouds (IRDCs Pérault et al. 1996; Egan et al. 1998). These clouds are observed in silhouette against the bright infrared background emission of the galactic plane. IRDCs are typically cold $(<25 \mathrm{~K})$ and dense $\left(\sim 10^{5} \mathrm{~cm}^{-3}\right)$ with masses ranging from $\sim 100-10^{5} M_{\odot}$ (Egan et al. 1998; Carey et al. 1998, 2000) and they are located at larger distances $(>1 \mathrm{kpc})$ compared to wellstudied low-mass star forming regions. A recently published catalog of dark clouds extracted from the GLIMPSE Survey by Peretto \& Fuller (2009) shows that most IRDCs have column densities below $N\left(\mathrm{H}_{2}\right) \approx 5 \times 10^{22} \mathrm{~cm}^{-2}$, with some extreme objects reaching $N\left(\mathrm{H}_{2}\right)>10^{23} \mathrm{~cm}^{-2}$. This new class of dark clouds are believed to represent the very early stages of clustered star formation, hence commonly referred to as cluster-forming clumps (e.g., Rathborne et al. 2006).

While most of the star-forming activity in the Galaxy takes place in the inner spiral arms and the molecular ring, significant activity also occurs beyond the solar circle $\left(R_{\mathrm{g}}>8.5 \mathrm{kpc}\right)$. Prominent examples include well studied nearby $(<500 \mathrm{pc})$ regions such as the Orion and Taurus-Perseus-Auriga complexes 
as well as more distant ( $>2 \mathrm{kpc}$ ) active star forming regions such as W3 and NGC 7538. However, the outer Galaxy is generally somewhat neglected in studies of star formation compared with the inner Galaxy, which is unfortunate because it is a useful laboratory to study the effect of different external conditions on the process that initiates stellar birth. In particular, the metallicity, pressure, and radiation field in the outer Galaxy are considerably different from the inner Galaxy (Brand \& Wouterloot 1995; Rudolph et al. 2006), which makes it by far the most nearby region to study the effect of such variations on star formation.

The aim of this work is to locate star-forming regions in the outer galactic plane. In order to find concentrations of molecular gas in the outer galactic plane, where the mid-infrared background is insufficient to measure absorption, we use the extinction to background stars. The traditional method to do this is via star counts (e.g., Wolf 1923), which is limited by extinction to nearby clouds, especially when using optical data. This problem can be avoided by using near-infrared data, but pick-up of emission from embedded objects makes this method unreliable.

An alternative to star counts is to use the near-infrared colour excess (NICE, Lada et al. 1994) which is widely used to map the extinction toward dark clouds (e.g., Cambrésy et al. 2002; Alves et al. 1998; Lada et al. 1999). However, this method relies on knowledge of the distance to the object, so that a correction for foreground stars can be made. The presence of foreground stars can reduce the measured color excess, and hence may influence an identification based on this, significantly (Lombardi 2005). Furthermore, identifying objects in an automated, uniform way toward large regions in the sky is difficult using NICE because the extinction law is not uniform in every direction. Also, an average reference color of background stars is required, which is usually adopted from a nearby, well selected, extinction-free region.

The identification of dark clouds by the extinction they impose upon background stars requires a method that:

1. determines the extinction from the colors of stars,

2. can be applied to small numbers of stars (for high resolution),

3. can be treated uniformly in all directions,

4. puts a confidence level to measurements,

5. is independent of foreground stars.

Because of the above mentioned limitations when using a nearinfrared color excess method, we have chosen to search for candidate dark cloud regions in a statistical manner rather than based on the absolute value of the extinction.

In two papers, we describe the identification process of candidate regions in the outer galactic plane with the goal to build up a sample of objects that can be used for future studies of (clustered) star formation throughout the Galaxy.

In this first paper we describe the Mann-Whitney U-test. This distribution-free statistical test can be applied uniformly to large data-sets in order to identify parts of the data with deviating properties. The purpose of this paper is to identify extended red regions in the sky using data from the Two Micron All Sky Survey (2MASS). In an accompanying paper (Frieswijk et al. 2010, hereafter Paper II) we perform a cross-correlation between the red regions presented here and existing data (optical dark clouds, MSX, IRAS and CO where available). The purpose of the second paper is to provide an indication of the actual nature of the reddening toward the identified regions

In Sect. 2 we summarize the 2MASS data and discuss why we use the $\left(H-K_{\mathrm{S}}\right)$ colors for the identification. In Sect. 3 we explain the Mann-Whitney U-test and describe the procedure that we follow in order to determine the statistics. In Sect. 4 we describe the friends-of-friends approach that is used to extract extended red regions from the reliability images produced by the U-test. In Sect. 5 we present the results. A catalog of the extended red regions is available online ${ }^{1}$ and at the CDS. The reliability images of the entire outer galactic plane are made available in FITS format from the same website. The final section summarizes our main conclusions.

\section{Data: the two micron all sky survey}

The two micron all sky survey (2MASS) scanned the entire sky uniformly in three near-IR bands: $J(1.25 \mu \mathrm{m}), H(1.65 \mu \mathrm{m})$ and $K_{\mathrm{S}}(2.17 \mu \mathrm{m})$. The $2 \mathrm{MASS}$ Point Source Catalog (2MASS PSC, Skrutskie et al. 2006) consists of accurate positions (astrometric accuracy rms $<200$ mas) and brightness information for over 400 million stars. The point source catalog is $>99 \%$ complete up to $J<15.8, H<15.1$ and $K_{\mathrm{S}}<14.3 \mathrm{mag}$. The photometrical signal-to-noise ratio is $>10$ (i.e. $\sigma_{J, H, K_{\mathrm{s}}} \lesssim 0.1 \mathrm{mag}$ ) for sources brighter than the above stated completeness limits. The so-called faint extension of the point source catalog includes sources that reach 0.5 to $1.0 \mathrm{mag}$ beyond the above limits. The completeness, reliability and uniformity of the faint extension sources are not as good as the high-reliability PSC and photometric errors increase up to $\sigma_{J, H, K_{\mathrm{s}}} \sim 0.4 \mathrm{mag}$. We make use of all available sources in 2MASS, including the faint extension. This increases the total number of objects by almost a factor 2 compared to star count studies, where the faint extension cannot be used because the data are incomplete.

The $\left(H-K_{\mathrm{S}}\right)$ color is an appropriate choice to perform the identification of red regions because the intrinsic $\left(H-K_{\mathrm{S}}\right)$ color of stars spans only a narrow range. For spectral types A0V to M6III the range is about 0.00 to $0.30 \mathrm{mag}$ (Bessell \& Brett 1988; Wainscoat et al. 1992). In the Solar neighbourhood the average $\left(H-K_{\mathrm{S}}\right)$ color is about $0.13 \mathrm{mag}$ with a standard deviation of $0.1 \mathrm{mag}$. This assumes no reddening due to extinction and takes into account the sensitivity limit of 2MASS (14.3 mag for $K_{\mathrm{S}}$-band). By including the faint extension, the average $\left(H-K_{\mathrm{S}}\right)$ color increases slightly $(0.15 \mathrm{mag})$ due to faint, intrinsically redder M-dwarfs. If a region appears significantly redder, this suggests that either foreground extinction is present or the intrinsic color distribution of stars in that direction is different. The latter can be important if for example a group of young stellar objects, which are intrinsically redder, is located in the observed field. Though such regions will be identified being intrinsically red rather than reddened, they still represent star forming regions. A discrimination between the two, which is not obvious from the work in this paper, can be done by looking at other available data and will be discussed in Paper II.

We analyse the 2MASS data in the outer Galaxy from $l=$ $90^{\circ}$ to $270^{\circ}$ and $b=-3.5^{\circ}$ to $+3.5^{\circ}$. In order to process the data on a desktop computer, we divided the area up in regions of $2^{\circ} \times 2^{\circ}$, with a sampling separation between different fields of one degree.

\section{Method}

\subsection{The Mann-Whitney U-test}

The goal of this project is to identify, based on near-IR $\left(H-K_{\mathrm{S}}\right)$ colors, those regions which are redder compared to the background. One potential approach is to calculate an average color

1 http://www.astro.rug.nl/ ismgroup/OuterGalaxy/ 
of a few stars and test whether that average is different than a background sample of stars. This approach has some significant drawbacks: how many sample stars should be used and what is the stellar color of the background given that this value changes over the Galactic Plane? Is the average the correct statistic to calculate? Why not use the median or some other statistic? Furthermore, the distribution of the colors of stars is not a standard parametric distribution. It is definitely not a Normal distribution which is needed if we want to use the average colors of stars and calculate a confidence based on a standard deviation. What is needed is a statistic, which does not depend on the distribution of stellar colors and which works when comparing small samples against a background of many stars in a robust manner.

The Mann-Whitney U-test (also referred to as the Wilcoxon rank-sum test, hereafter referred to as U-test) is a well-known non-parametric significance test first proposed by Wilcoxon (1945) and extended to arbitrary sample sizes by Mann \& Whitney (1947). The statistic is based on the rank of the observation and not the observation value itself. This makes it a non-parametric statistic. The test is used to assess whether two samples of observations, say sample $A$ and $B$, come from the same distribution. Samples $A$ and $B$ are required to be independent and the observations should be ordinal or continuous measurements. The U-test is hypothesis testing, where the hypothesis is that the two samples come from the same distribution. This is called the Null hypothesis. The statistic which is calculated is the sum of the joint ranking $(U)$ of all the observations, in our case the $\left(H-K_{\mathrm{S}}\right)$ colors.

\section{An example}

The distribution of the sum of ranks of $A$ and $B$, is readily calculated for samples drawn from the same parent distribution. Take the case of 3 observations against 3 other observations. If the observations are truly randomly drawn from the same parent distribution, then one would expect a lower chance of pulling the three highest values for sample $A$ (ranks $4,5,6$ ) and $B$ therefore resulting in the three lowest ranks $1,2,3$. One would rather expect a more random mix, e.g., 1, 4, 6 and 2,3,5. In the example above the sum of the ranks in the first case, $U_{A}=15, U_{B}=6$ is less likely than the second sum of ranks, $U_{A}=11, U_{B}=10$. In this way the distribution of rank sums is built up.

If the rank sum statistic is $U_{A}=15$, the probability of this occurring randomly is small (5\%), which means the Null hypothesis can be rejected on a statistical basis to a particular level of confidence (95\%), in favor of the alternative or test hypothesis that the $A$ samples are larger than the $B$ samples. The alternative hypothesis must be created in such a way that it is the only alternative to the Null hypothesis. Rejecting the Null hypothesis means that the alternative hypothesis must be accepted at that level of confidence.

The U-test can be applied to large scale surveys in order to identify regions where source properties deviate significantly with respect to a reference field. We use the U-test statistic to test whether a set of colors of stars is different than the set of colors of stars from another sample. We test against the Null hypothesis that the set of colors is actually the same. When the test rejects the Null hypothesis (to a certain confidence level), we must accept the test hypothesis that the colors are actually different. As can be seen from the example above, there is a fundamental difference if $U_{A}$ is greater or less than $U_{B}$. For near-IR colors this means we could test for stars redder or bluer than the background stars.

For this work, we want to compare as few stars as possible against a background of tens of thousands. The benefit of using a non-parametric test is that whatever size the two samples have, the distribution of the U-test statistic is known from pre-calculated tables (e.g., Wall \& Jenkins 2003), unlike for a parametric test. However, tables for finding the probabilities of $U$ usually do not contain values for samples with size in excess of 20. For large sample sizes though, the distribution will approach a Normal distribution thanks to the Central Limit Theorem. The mean and standard deviation of the variables is simply given by the mean and standard deviation of the Normal distribution. Moses (1964) stipulates that the smallest number of samples is 5, to still be able to use the Normal approximation for the U-test. For a parametric test such as the average, a Normal approximation is only valid for higher sample sizes. This is evaluated in Sect. 3.5.

\subsection{Applying the U-test procedure}

We perform our calculations on a regular grid. For each gridcell the distribution of properties, i.e., star colors, is referred to as sample $A$. A reference field represents sample $B$ and is determined locally. The choice of the reference field is explained in Sect. 3.3. The size of the grid-cells can be chosen at will, but a minimum number of sources (5; see Sect. 3.5) is required to retrieve a reliability based on the Normal approximation. The choice of the resolution is explained in more detail in Sect. 3.4.

The following steps are involved in the calculation of the probability $P$ for any given grid-cell, where $P$ is the probability that a cell contains a redder color distribution compared to the reference field. The whole procedure is schematically given also in Fig. 1.

1. Let $A$ be the distribution of colors in the cell (with $m$ members) and $B$ the local reference distribution (with $n$ members, where $n$ is very large in this study, i.e., $\sim 10^{4-5}$ ). The Null hypothesis $\left(H_{0}\right)$ is that $A$ and $B$ have the same parent population, or in perspective of our work, that the parent population of $A$ is given by $B$. Note that when $m$ is less than 5 , the probability $P$ is set to $50 \%$ and the next cell is processed.

2. Rank the colors in ascending order for the combined members of $A$ and $B$. Preserve the $A$ or $B$ identity for each member.

3. Sum the number of $A$-rankings to get the statistical value $U_{A}$.

Generally, numerically equivalent (also known as tied) observations are assigned with the average of their respective ranks. However, we avoid tied observations by adding very small random numbers to the color values $\left(<10^{-4}\right)$. This has no impact on the results, but makes the computations easier.

For large samples, the sampling distribution tends to a Normal distribution (see also Sect. 3.5) with a mean $\mu_{A}$ and variance $\sigma_{A}^{2}$ given by

$\mu_{A}=m(N+1) / 2$ and $\sigma_{A}^{2}=m n(N+1) / 12$,

respectively, where $N=m+n$. The significance, $z$, can then be assessed from the Normal distribution by calculating

$z=\frac{U_{A}-\mu_{A} \pm 0.5}{\sigma_{A}}$.

The term \pm 0.5 , in statistics often referred to as the continuity correction factor, is a correction required to maintain continuity when a discrete distribution, such as the U-test statistic, is approximated by a continuous distribution, such as the Normal distribution. Because the Normal distribution consists of all real numbers, but the U-test values are discreet (integer values), a 


\section{1) Define $A$ and $B$}

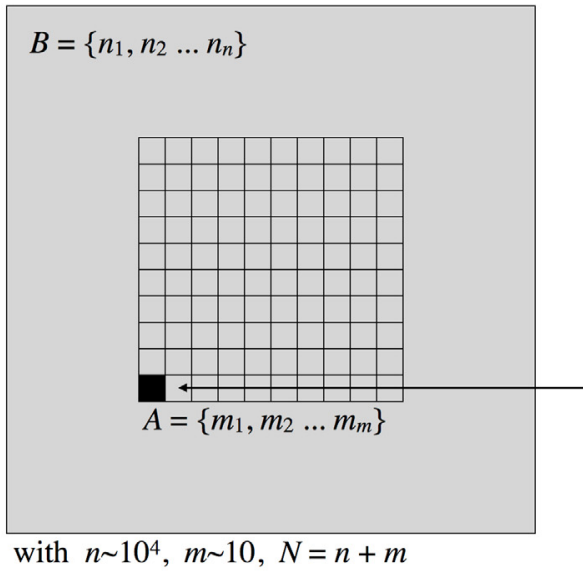

2) Order and rank members of $A$ and $B$

$$
\begin{gathered}
n_{2}<m_{1}<n_{5}<m_{\mathrm{m}}<n_{n-1}<n_{1}<m_{3}<n_{n} \ldots \\
\operatorname{ranks}_{A, B}=\left\{1_{B}, 2_{A}, 3_{B}, 4_{A}, 5_{B}, 6_{B}, 7_{A}, 8_{B} \ldots\right\}
\end{gathered}
$$

3) Calculate statistics

$$
U_{A}=\Sigma\left(\operatorname{ranks}_{A}\right)
$$

and use equations 1,2 and 3

\section{Process next cell}

Fig. 1. Schematic representation of the U-test procudure.

Normal approximation should identify the discreet event " $n$ " with the normal interval " $(n-0.5, n+0.5)$ ", where $n$ is any integer value. In this situation we use -0.5 (instead of +0.5 ) because we consider only objects in the upper tail, i.e., where the distribution is redder (instead of bluer) than the reference distribution. The probability $P$ is calculated by evaluating the integral from -inf to $z$ of the Gaussian probability density function $\Phi(z)$, where $\Phi(z)$ is given by

$\Phi(z)=\frac{1}{\sqrt{2 \pi}} \mathrm{e}^{-z^{2} / 2}$.

\subsection{Reference distribution}

A single reference distribution is almost certainly not representative for every field along the galactic plane. First, because the stellar population, and thus the color distribution, may vary with galactic coordinates. Second, because foreground extinction will be present everywhere. The reddening caused by the extinction depends on the amount of foreground material and on the dust properties toward different lines of sight. Although the overall extinction features are interesting, the target for this work is to find extreme color changes relative to the local environment. Therefore we make no attempt to extract large scale extinction features along the galactic plane.

Instead of using a single reference distribution everywhere, we define the distribution of colors in every $2^{\circ} \times 2^{\circ}$ field as a reference for the grid cells in the inner square degree of the respective field. This means that we select a local reference distribution as best representation for the colors in each field, thus avoiding the effect of color variations on galactic scale. Note that the colors of stars in the cells that are being compared to the local reference are omitted from the reference distribution itself.

\subsection{Resolution limitation}

The limiting factor for the spatial resolution that can be achieved in performing the U-test is determined by the minimum of 5 sources required for the Normal approximation. As a requirement for the grid-cell size (resolution) we state that in every field at least $75 \%$ of the cells should contain 5 or more sources. Because the stellar surface density decreases toward the galactic anti-centre $\left(l=180^{\circ}, b=0^{\circ}\right)$, we process the data at two different resolutions. The highest possible resolution, mostly applicable towards the spiral arm regions at $l \sim 90^{\circ}-140^{\circ}$ and $l \sim 240^{\circ}-270^{\circ}$, is $60^{\prime \prime}$. For the region between $l \sim 140^{\circ}-240^{\circ}$ we require a grid with cells of $90^{\prime \prime}$. Note that we process the entire outer galactic plane at both resolutions and all data are made available. The average number of stars is about 8 per cell for the high resolution grid toward the spiral arm regions as well as for the low-resolution grid toward the anti-center region.

\subsection{Minimum requirements: a Monte Carlo approach}

In the U-test we assume that even for small samples drawn from a large set of sources, the distribution of the significance values $(z)$ tends to Normal. With a Monte Carlo simulation we show that the significance distribution of randomly drawn samples from a large distribution is indeed close to a Gaussian probability density distribution. We specifically simulate samples of 8 sources because this is the average number of sources that are present in a grid cell. The large distribution is extracted from an observed field centered on $l=122^{\circ}, b=1^{\circ}$. We have performed the same simulation using various other fields to test whether the results below change when less, or more reddening is present in a field. We find that this is not the case.

The resulting distribution of $z$ is given in the lower left panel in Fig. 2. In the upper left panel we display the distribution of $z$ for samples of 5 sources, the minimum sample size for a proper U-test result according to Moses (1964). The peak as well as the wings are slightly overestimated by the Gaussian profile in the simulation for samples of 5 sources, but increasing to samples of 8 shows that the simulation converges fast to a Normal distribution. The difference between the the areas under the curves for the wing region $(z>2)$ is less than $10 \%(<5 \%$ for samples of 8$)$. By assuming that the profile is given by a Gaussian distribution, we underestimate the actual probability of having a red pixel in the regime considered here, i.e., $z \geq 2.326$ corresponding to a probability $P \geq 99 \%$ when evaluating the integral over Eq. (3).

We compared the simulated results of the U-test with two parametric tests: the average and median. The right panels of Fig. 2 show that the distribution of average values deviates much more from a Gaussian profile compared to the U-test. The distribution is clearly skewed and cannot be represented by a simple profile. The area in the wing $\left(H-K_{\mathrm{S}}>0.5 \mathrm{mag}\right)$ is underestimated by $\approx 50 \%(\approx 100 \%$ for 8 samples). The shape of the distribution of average values approaches a Normal distribution only for sample sizes $\gtrsim 20$, but the area in the wing remains underestimated. Almost identical results are found for the distribution of median colors, with a comparably large discrepancy in the wing area. 
W. W. F. Frieswijk and R. F. Shipman: Extended red regions in the outer galactic plane
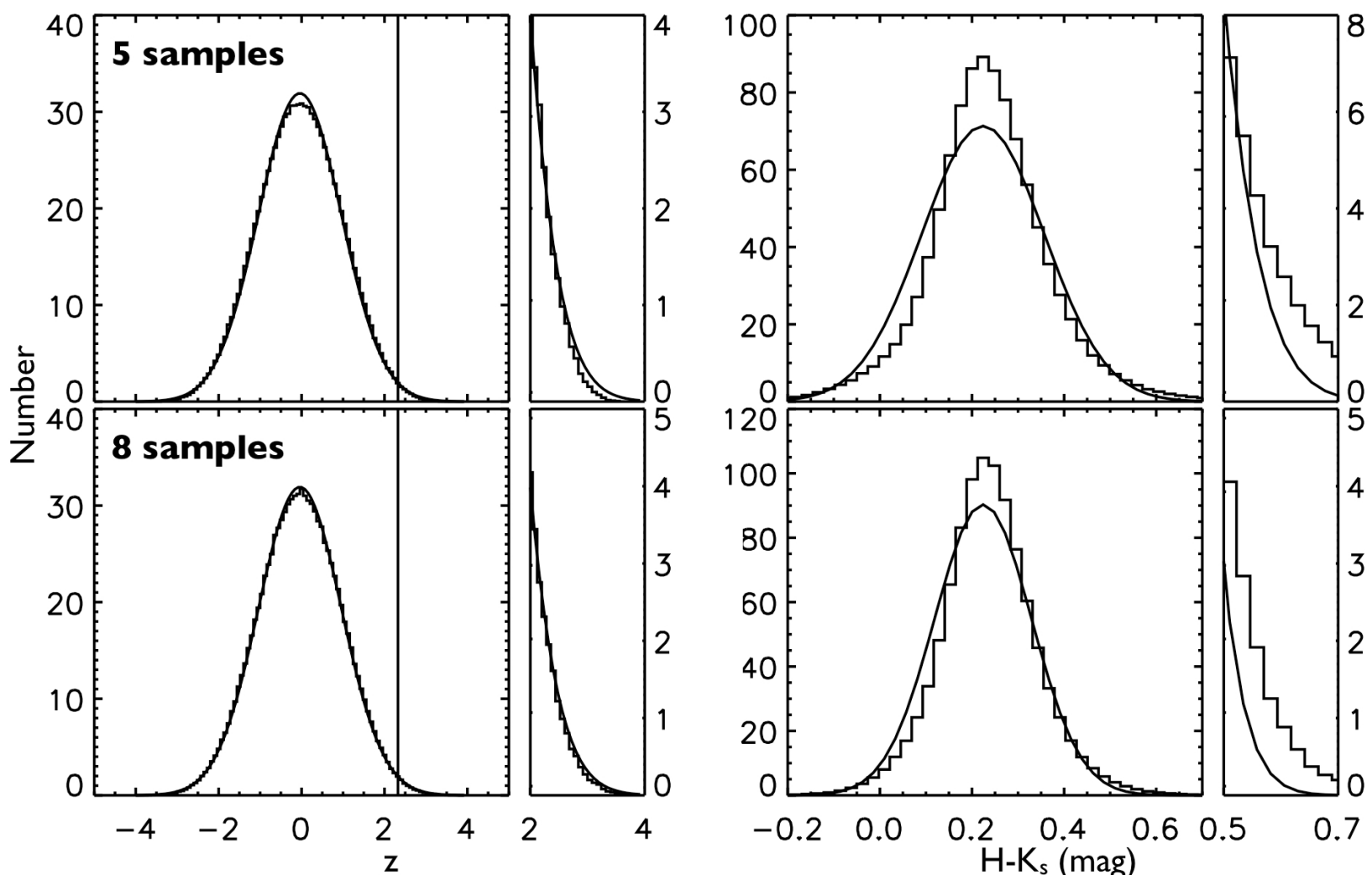

Fig. 2. Visualization of the result of a Monte Carlo simulation, where we randomly draw 6000 samples of 5 (upper histograms) and 8 (lower histograms) 2MASS colors from a test field at $l=122^{\circ}, b=1^{\circ}$. The distributions of the significance $z$ are displayed on the left. The right histogram displays the distribution of average colors in the same simulation. A blow-up of the high-end tail of each distribution is given in the narrow panels right of the histograms. The solid lines in each panel represent Gaussian profiles where the peak position and FWHM are adopted from the simulated distributions.

The average and median colors are often used, e.g., for nearinfrared color excess (NICE). Even though the median value is less sensitive to (extreme) outliers in the distribution than the average, the main issue is, that both are not well represented by simple profiles. This makes it difficult, if not impossible, to put a significance to the measured values. This justifies the use of the non-parametric U-test statistics for the automated identification process presented in this paper.

\subsection{Reliability and completeness}

There are several considerations that need to be kept in mind when interpreting the resulting images created by the U-test method. These are related to the reliability and completeness of the cells that are identified as being red. We discuss them here.

\section{Type I error}

The first error is related to the reliability, or the significance level of the selected cells. If, as in our case, rejection is defined at $99 \%$ this suggests that about $1 \%$ of the cells will be selected on statistical arguments, rather than being red. However, these cells are expected to be randomly distributed over the fields. To exemplify that this is indeed the case, we consider the following values extracted from the low-resolution $2^{\circ} \times 2^{\circ}$ field toward $l=166^{\circ}, b=1^{\circ}$. This field is assumed to be empty, because no objects, identified as clusters of red cells, ended up in a final identification. However, the field contains 76 cells out of a total of $6561(\approx 1 \%)$ where the Null hypothesis of the U-test was rejected. Indeed, these cells are spread more or less randomly over the field, or else they would have been identified as clustered objects.

\section{Type II error}

This error is related to the cells that are not identified, i.e., where the Null hypothesis is not rejected but should have been. It is very difficult to put a realistic value to this error. In a broad sense it is inversely related to the Type I error. That is, the more reliable the sample is the less complete it is and vice versa. Furthermore, the Type II error usually occurs when sample sizes are too small. Because we have chosen to produce a high reliability catalog, we are giving in on completeness due to this error.

\section{Sample statistics}

According to Moses (1964), when one of the distributions that go into the U-test contains fewer than 5 sources the outcome of the U-test is unreliable. There is not sufficient information available on the color distribution. Cells containing fewer than 5 sources are omitted in the identification process and, thus, introduce another form of incompleteness which depends on the resolution. Processing at higher resolution means being less complete.

Considering the limitations and reliability of the method, we have decided to put the effort in producing a reliable target list at relatively high resolution $\left(60^{\prime \prime}\right.$ and $\left.90^{\prime \prime}\right)$, rather than being complete. Furthermore, our intention is not to evaluate the content or structure of the outer galactic plane nor finding all 

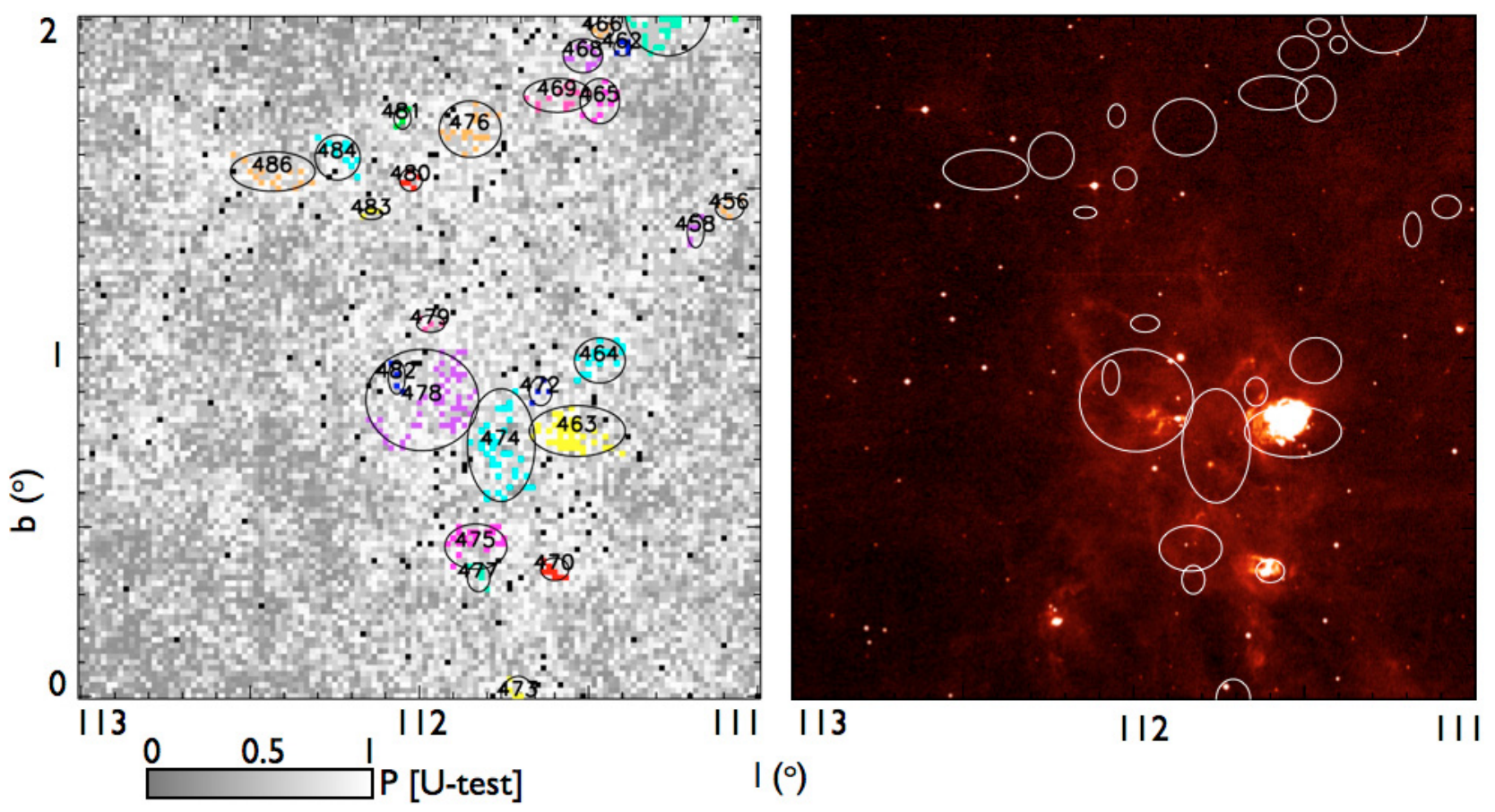

Fig. 3. The left panel displays the probability image returned by the U-test. The grey-scales represent the probability that the color distributions in the cells are redder with respect to the reference distribution. The friends-of-friends method identified the extended red regions, displayed in arbitrary colors. The ellipses give the spatial extent of the regions. Black cells are identified as being red, but are considered isolated. Objects 463 and 470 correspond to NGC 7538 and S 159, respectively. Object 474 is the first outer Galaxy "cluster-forming clump"-candidate, identified as such by the work presented here. The right image shows the same region in MSX $8 \mu \mathrm{m}$ emission for comparison. Bright emission from warm dust, heated by embedded, massive young stars, is seen toward both NGC 7538 and S 159. The upper part of the image is almost devoid of extended $8 \mu \mathrm{m}$ emission, typical for the majority of directions in the outer Galaxy.

"dark clouds" present in the outer Galaxy. We purely present a statistical method that enables the identification of real objects.

\section{Extracting candidate sources}

Friends-of-friends approach

To increase the reliability of red regions and to avoid the selection of random cells, we add an additional step to the process. By means of a friends-of-friends algorithm, we select only clusters of 4 or more red cells for the final list of candidate objects. The reason for using 4 cells here is based on the probability of identifying clusters of randomly distributed red cells, which becomes negligible ( $\ll 1 \%)$ for groups larger than 3 cells. These probabilities depend on the linking length described below and were evaluated in the Monte Carlo simulations. The friends-of-friends algorithm, first applied by Huchra \& Geller (1982) and often encountered in cosmological studies, is a well-known technique and can be simplified to find groups, or structures, in projection on the sky. We use the friends-of-friends approach to identify groups of cells on the sky where the color distribution of stars is red compared to the local surroundings, i.e., where the U-test returns a probability of $P=99 \%$ or higher.

\section{Linking length}

A crucial parameter required for the friends-of-friend method is the linking length, $L$, used to determine whether selected cells belong to the same group or not. If $L$ is taken too large, all cells will be assigned to the same group. If $L$ is too small all cells will be considered isolated. Because we do not know the cause of the red color distribution in different cells (either extinction due to foreground material at unknown distance, or a concentration of intrinsically red objects at unknown distance) it is impossible without further information to put a physical size to $L$. We therefore choose a linking length in terms of the cell size, $s_{\text {cell }}$.

An upper limit for $s_{\text {cell }}$ can be estimated from an empty field. Consistent with the defined reliability, about $1 \%$ of the cells in an empty field are rejected by the U-test and spread randomly over the field. We assess an average distance between rejected cells by using a Monte Carlo simulation, where $N$ cells $\left(=1 \%\right.$ of $\left.a^{2}\right)$ are selected at random locations from a square box with side $a$. For $a^{2}$ we use the number of cells that make up a $2^{\circ} \times 2^{\circ}$ field at the given resolutions, i.e., $a=81$ cells at $90^{\prime \prime}$ and $a=121$ cells at $60^{\prime \prime}$ resolution. The average distance in the simulation converges to 5.3 and 5.2 times the cell-size, respectively. This is in agreement with the theoretically expected value, given by

average distance $=\frac{1}{2} \sqrt{\frac{a \times a}{N}}=5 \quad\left[\times \mathrm{s}_{\text {cell }}\right]$

(Hertz 1909; Clark \& Evans 1954).

A linking length much larger than this average distance will group cells together even if the distribution is random. This obviously contradicts the goal of finding clustered regions. A lower limit is obviously the cell size itself. We have tested the method by varying $L$ between 2 and 5 . The higher values $(>3)$ identify mostly large scale structures $\left(>0.5^{\circ}\right)$. For a value of 2 , most cells are isolated or grouped with fewer than 3 other cells. We decide to use a linking length half that of the average distance between random points, i.e., $L=2.5$ times the cell size, for both resolutions. The probability for finding "accidental groups" of 4 cells using $L=2.5$ and considering that $1 \%$ of the cells are randomly selected by the U-test was evaluated in the Monte Carlo simulations described above and is $\$ 0.1 \%$. 
W. W. F. Frieswijk and R. F. Shipman: Extended red regions in the outer galactic plane

Table 1. Excerpt from the catalog of extended red objects.

\begin{tabular}{ccccccc}
\hline \hline $\begin{array}{c}\text { FrSh } \\
(1)\end{array}$ & $\begin{array}{c}1 \\
(2)\end{array}$ & $\begin{array}{c}\mathrm{b} \\
(3)\end{array}$ & $\begin{array}{c}N_{\text {cell }} \\
(4)\end{array}$ & $\begin{array}{c}\Delta l \\
(5)\end{array}$ & $\begin{array}{c}\Delta b \\
(6)\end{array}$ & $\begin{array}{c}2 \mathrm{M} \\
(7)\end{array}$ \\
& $\mathrm{deg}$ & $\mathrm{deg}$ & & pix & pix & \\
\hline $\mathrm{H} 456$ & 111.08 & 1.44 & 5 & 5 & 4 & 43 \\
$\mathrm{H} 457$ & 111.27 & 2.02 & 41 & 15 & 15 & 299 \\
$\mathrm{H} 458$ & 111.18 & 1.38 & 5 & 3 & 6 & 49 \\
$\mathrm{H} 459$ & 111.20 & 2.17 & 6 & 3 & 5 & 51 \\
$\mathrm{H} 460$ & 111.22 & -0.83 & 5 & 3 & 5 & 79 \\
$\mathrm{H} 461$ & 111.27 & -0.75 & 4 & 3 & 5 & 68 \\
$\mathrm{H} 462$ & 111.40 & 1.92 & 5 & 3 & 3 & 38 \\
$\mathrm{H} 463$ & 111.53 & 0.78 & 40 & 17 & 9 & 655 \\
$\mathrm{H} 464$ & 111.47 & 0.99 & 16 & 9 & 8 & 122 \\
$\mathrm{H} 465$ & 111.47 & 1.76 & 9 & 7 & 8 & 67 \\
$\mathrm{H} 466$ & 111.46 & 1.97 & 4 & 4 & 3 & 29 \\
$\mathrm{H} 467$ & 111.48 & -0.66 & 5 & 5 & 2 & 78 \\
$\mathrm{H} 468$ & 111.52 & 1.89 & 10 & 7 & 6 & 70 \\
$\mathrm{H} 469$ & 111.59 & 1.77 & 13 & 12 & 6 & 80 \\
$\mathrm{H} 470$ & 111.60 & 0.38 & 8 & 5 & 4 & 123 \\
$\mathrm{H} 471$ & 111.62 & 2.17 & 4 & 6 & 2 & 24 \\
$\mathrm{H} 472$ & 111.64 & 0.90 & 4 & 4 & 5 & 32 \\
$\mathrm{H} 473$ & 111.71 & 0.00 & 8 & 6 & 7 & 84 \\
$\mathrm{H} 474$ & 111.76 & 0.74 & 34 & 12 & 20 & 335 \\
$\mathrm{H} 475$ & 111.83 & 0.44 & 20 & 11 & 8 & 162 \\
$\mathrm{H} 476$ & 111.85 & 1.67 & 16 & 11 & 10 & 122 \\
$\mathrm{H} 477$ & 111.82 & 0.35 & 5 & 4 & 5 & 46 \\
$\mathrm{H} 478$ & 111.99 & 0.88 & 52 & 20 & 18 & 468 \\
$\mathrm{H} 479$ & 111.97 & 1.10 & 4 & 5 & 3 & 31 \\
$\mathrm{H} 480$ & 112.03 & 1.52 & 5 & 4 & 4 & 53 \\
$\mathrm{H} 481$ & 112.05 & 1.71 & 4 & 3 & 4 & 29 \\
$\mathrm{H} 482$ & 112.07 & 0.94 & 4 & 3 & 6 & 34 \\
$\mathrm{H} 483$ & 112.14 & 1.42 & 4 & 4 & 2 & 41 \\
$\mathrm{H} 484$ & 112.24 & 1.59 & 11 & 8 & 8 & 101 \\
$\mathrm{H} 485$ & 112.32 & 2.84 & 7 & 5 & 6 & 51 \\
$\mathrm{H} 486$ & 112.43 & 1.55 & 15 & 15 & 7 & 109 \\
\hline & & & & & &
\end{tabular}

A consequence of setting the linking length to 2.5 is that groups are identified with cells in between the red ones that may not be identified as red. This can either be due to a type II error (Sect. 3.6), or insufficient stars present in that cell (the sample statistics, Sect. 3.6). In both cases this illustrates that not all reddened regions can be detected and thus implies that we are limited in the completeness of the catalog.

\section{Results}

Figure 3 displays an example of the output of the U-test in the form of a probability image for the region centered on $l=112^{\circ}$, $b=1^{\circ}$. For comparison we display the $8 \mu \mathrm{m}$ emission as observed by MSX. We identify 24 extended red regions in this field. The red cells of these regions are presented in different colors in Fig. 3, with the spatial extent of the objects indicated by the ellipses. Some objects clearly correlate with extended bright $8 \mu \mathrm{m}$ emission coming from well-known star forming regions such as NGC 7538 or S 159. This field also contains object H474, which has been selected as a "massive dark cloud"-candidate for follow-up observations. These observations have revealed the first infrared dark cloud identified as such in the outer galactic plane (Frieswijk et al. 2007, 2008).

The resulting catalog of extended red regions and the probability images in FITS-format are available online ${ }^{2}$. The low-resolution part consists of 1589 objects and the high resolution part of 1320 . The catalog is available in electronic

\footnotetext{
${ }^{2}$ http://www . astro.rug.nl/ ismgroup/OuterGalaxy/
}

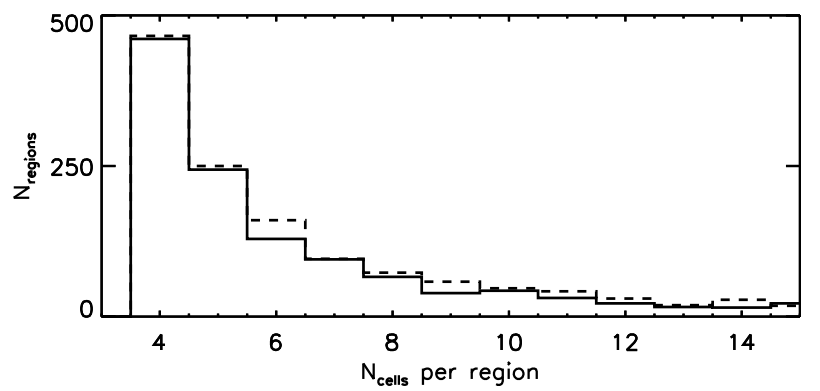

Fig. 4. Histogram displaying the distribution of the number of cells per extended red region for the high-resolution (solid) and low-resolution (dashed) catalog.

form at the CDS with sources designated FrSh LNNNN and FrSh HNNNN for low- and high resolution objects, respectively. Table 1 presents an excerpt from the high resolution catalog. The objects in the table correspond to those that are identified in the field displayed in Fig. 3 and include NGC 7538 (FrSh H463) and S 159 (FrSh H470). The different columns present the following information:

Column (1): Object identification,

Columns (2)-(3): Galactic coordinates of the object centre,

Column (4): Total number of red pixels,

Columns (5)-(6): Extent in $l$ and $b$ in pixel units (1 pix $=1^{\prime}$ ), Column (7): Number of 2MASS sources.

Note that the number of 2MASS sources in Col. (7) contains information on the stellar surface density. In principle this can be used to distinguish between objects identified by extinction and objects representing a clustering of young stars, because extinction may lower the stellar density whereas a clustering may increase it. However, because we are using the 2MASS catalog including the faint extension the variations in stellar surface density may also arise from the incompleteness of the catalog and we do not discuss it further in this paper.

\subsection{Size- and spatial distribution}

The linear extent of the extended red regions ranges from a few arc-minutes to about a degree. Figure 4 gives an overview of the cell-number distribution for the regions in the high- and low resolution catalog. This shows that about half of the regions are identified as groups of 4 or 5 cells. Regions consisting of more than 10 cells account for $18 \%$ of the objects in the high resolution catalog and for $28 \%$ of the objects in the low resolution one.

Figure 5 shows the sky distribution of the extended red regions in the outer galactic plane. The solid histogram in the lower panel represents the number of high-resolution regions in bins of $5^{\circ}$ galactic longitude. The dashed histogram displays the distribution of low-resolution regions. The galactic latitude distribution is given in the horizontal histogram in the right panel. Several authors have found that the distribution of (molecular) clouds toward the inner galactic plane peaks at negative galactic latitude (Peretto \& Fuller 2009; Schuller et al. 2009; Rosolowsky et al. 2010), suggested to result from the Sun being located slightly above the Galactic plane. We do not find a similar distribution toward the outer Galaxy. In fact, about $60 \%$ of the high resolution objects are at positive latitudes $(\approx 50 \%$ for low resolution). However, this can be attributed to the local, 


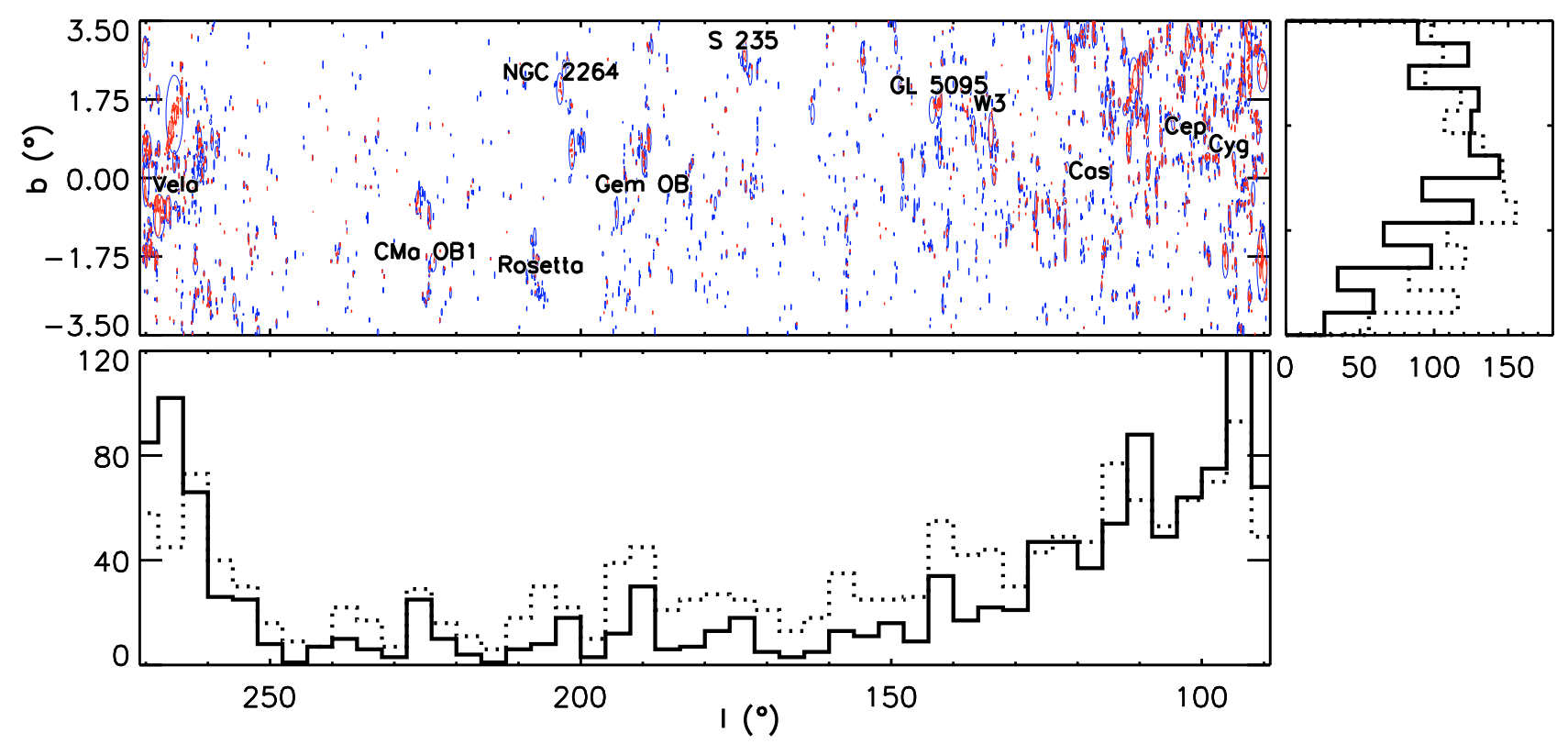

Fig. 5. Spatial distribution of extended red regions identified in the outer galactic plane at 60" (solid histogram, red regions) and 90" (dashed histogram, blue regions) resolution. The regions are identified with a reliability of $>99.9 \%$. A few well-known star forming regions in the Galaxy are given for reference.

well-populated, Vela and Cygnus/Cepheus star forming regions which are located mainly above the plane.

The 2-dimensional spatial distribution is displayed in the upper panel. The red and blue ellipses display the location and linear extent of the high and low resolution regions, respectively. Some well-known areas associated with molecular clouds and star forming activity in the outer Galaxy are indicated. Most of the regions we find are well confined within the extent of these large complexes, and by far most regions are identified in the crowded spiral arm regions near Vela and Cygnus/Cepheus. Toward lower stellar densities $\left(l \approx 140^{\circ}-240^{\circ}\right)$ there are more regions identified with the low resolution than the high resolution grid because at high resolution there are often insufficient stars in a cell to use for the U-test. Many of the large-scale regions identified at low resolution, in particular visible toward the Vela and Cygnus/Cepheus regions, are identified as groups of smaller regions with the high resolution grid.

\subsection{Column density sensitivity}

The objects in the catalog are identified without using a parameter that relates to a physical quantity. However, we do have information on the average $\left(H-K_{\mathrm{S}}\right)$ colors of cells in all objects and we use it to derive a rough estimate of the column density sensitivity. Note however, that we can not account for any corrections due to contamination of unreddened foreground stars and the values below represent only lower limits.

The average color in the cells of identified regions is $\langle(H-$ $\left.\left.K_{\mathrm{S}}\right)\right\rangle \approx 0.54 \mathrm{mag}$ with a standard deviation of $\sigma \approx 0.18 \mathrm{mag}$. Some cells have an average color up to $\approx 2 \mathrm{mag}$. These values can be converted to a visual extinction $A_{\mathrm{V}}$ using the following equations;

$A_{\mathrm{V}}=15.9 \times E\left(H-K_{\mathrm{S}}\right)$

(Rieke \& Lebofsky 1985),

$E\left(H-K_{\mathrm{S}}\right)=\left(H-K_{\mathrm{S}-\text { observed }}\right)-\left(H-K_{\mathrm{S}-\text { intrinsic }}\right)$, where $E\left(H-K_{\mathrm{S}}\right)$ represents the color excess, $\left(H-K_{\mathrm{S}-\text { observed }}\right)$ the observed color in a cell and $\left(H-K_{\mathrm{S} \text {-intrinsic }}\right)$ the intrinsic color. For the intrinsic color we use a value of 0.15 mag (Sect. 2). This means we are sensitive to visual extinction values ranging from a few to $\approx 30 \mathrm{mag}$, corresponding to molecular column densities up to $N\left(\mathrm{H}_{2}\right) \approx 3 \times 10^{22} \mathrm{~cm}^{-2}$ (Bohlin et al. 1978). This is comparable to values found for the majority of IRDCs (Peretto \& Fuller 2009).

\section{Conclusions}

We have used a statistical approach in combination with a friends-of-friends algorithm to measure deviations in the spatial $\left(H-K_{\mathrm{S}}\right)$ color distribution of stars in the outer galactic plane, with the goal to identify extended reddened regions. We processed the galactic plane at $60^{\prime \prime}$ and $90^{\prime \prime}$ resolution, resulting in the identification of 1320 high resolution and 1589 low resolution extended red regions. The reliability of individual red cells that make up each object is $99 \%$ and by using a friendof-friends approach, the resulting catalog of objects is $99.9 \%$ reliable. Because we have put the effort in producing a highly reliable source list we cannot quantify the completeness of the catalog.

The majority of the objects are located toward well-known molecular cloud complexes and some correspond to specific, well-known objects such as NGC 7538 and S 159. This correlation strengthens the argument that the red nature of the regions is caused by extinction and/or embedded young stellar objects rather than intrinsic star color variations. The main goal of our analysis is to find previously undetected dark clouds in the outer Galaxy. However, many other types of objects are included in the catalog and the results may be compared to other outer Galaxy studies in the future.

Based on the parameters derived from the statistical test it is impossible to determine the nature of the reddening. The next step is to cross-correlate the objects with existing optical, infrared and $\mathrm{CO}$ data. This process is described in an accompanying paper (Frieswijk et al. 2010). 
W. W. F. Frieswijk and R. F. Shipman: Extended red regions in the outer galactic plane

Acknowledgements. We thank the anonymous referee for his/her care- ful reading of the manuscript and his/her constructive remarks. We would also like to thank Marco Spaans and Floris van der Tak for their helpful discussions and suggestions which have improved this manuscript. This publication makes use of data products from the Two Micron All Sky Survey, which is a joint project of the University of Massachusetts and the Infrared Processing and Analysis Center/California Institute of Technology, funded by the National Aeronautics and Space Administration and the National Science Foundation.

\section{References}

Alves, J. ., Lada, C. J., Lada, E. A., Kenyon, S. J., \& Phelps, R. 1998, ApJ, 506, 292

Bessell, M. S., \& Brett, J. M. 1988, PASP, 100, 1134

Bohlin, R. C., Savage, B. D., \& Drake, J. F. 1978, ApJ, 224, 132

Brand, J., \& Wouterloot, J. G. A. 1995, A\&A, 303, 851

Cambrésy, L., Beichman, C. A., Jarrett, T. H., \& Cutri, R. M. 2002, AJ, 123, 2559

Carey, S. J., Clark, F. O., Egan, M. P., et al. 1998, ApJ, 508, 721

Carey, S. J., Feldman, P. A., Redman, R. O., et al. 2000, ApJ, 543, L157

Clark, P. J., \& Evans, F. C. 1954, Ecology, 35, 445

Egan, M. P., Shipman, R. F., Price, S. D., et al. 1998, ApJ, 494, L199

Frieswijk, W. F., Spaans, M., Shipman, R. F., et al. 2008, ApJ, 685, L51

Frieswijk, W. W. F., Spaans, M., Shipman, R. F., Teyssier, D., \& Hily-Blant, P. 2007, A\&A, 475, 263
Hertz, P. 1909, Mathematische Annalen, 67, 387

Huchra, J. P., \& Geller, M. J. 1982, ApJ, 257, 423

Lada, C. J., Alves, J., \& Lada, E. A. 1999, ApJ, 512, 250

Lada, C. J., \& Lada, E. A. 2003, ARA\&A, 41, 57

Lada, C. J., Lada, E. A., Clemens, D. P., \& Bally, J. 1994, ApJ, 429, 694

Lombardi, M. 2005, A\&A, 438, 169

Mann, H. B., \& Whitney, D. R. 1947, Annals of Mathematical Statistics, 18, 50

Moses, E. L. 1964, Journal of the American Statistical Association, 59, 645

Pérault, M., Omont, A., Simon, G., et al. 1996, A\&A, 315, L165

Peretto, N., \& Fuller, G. A. 2009, A\&A, 505, 405

Rathborne, J. M., Jackson, J. M., \& Simon, R. 2006, ApJ, 641, 389

Rieke, G. H., \& Lebofsky, M. J. 1985, ApJ, 288, 618

Rosolowsky, E., Dunham, M. K., Ginsburg, A., et al. 2010, ApJS, 188, 123

Rudolph, A. L., Fich, M., Bell, G. R., et al. 2006, ApJS, 162, 346

Schuller, F., Menten, K. M., Contreras, Y., et al. 2009, A\&A, 504, 415

Shu, F. H., Adams, F. C., \& Lizano, S. 1987, ARA\&A, 25, 23

Skrutskie, M. F., Cutri, R. M., Stiening, R., et al. 2006, AJ, 131, 1163

Wainscoat, R. J., Cohen, M., Volk, K., Walker, H. J., \& Schwartz, D. E. 1992, ApJS, 83, 111

Wall, J. V., \& Jenkins, C. R. 2003, in Practical Statistics for Astronomers (Cambridge University Press)

Wilcoxon, F. 1945, Biometrics Bulletin, 1, 80

Wolf, M. 1923, Astron. Nachr., 219, 109

Zinnecker, H., \& Yorke, H. W. 2007, ARA\&A, 45, 481 